\title{
Gover Story
}

\section{IGHTER MARGINS AND TIGHTER BELTS, BUT STILL TREATING PATIENTS: THE VERDICT ON THE MEDICARE MODERNIZATION ACT}

ew oncologists were taken by surprise when the Medicare Modernization Act (MMA) became law on January 1, 2005; in fact, an informal poll found that many had already been preparing for it well in advance.

Now that the MMA has become law, JOP thought it would be beneficial to look at the real-world consequences on patients and practices. JOP also wanted to explore some of the strategies oncologists are using to adapt their practices.

Since there is no such thing as a "typical" oncology practice, JOP selected three as representative: a Kansas City, Kansas practice with 35 oncologists, an Albuquerque, New Mexico practice with 11, and one in Paducah, Kentucky with three oncologists. All three practices serve commercial and Medicare patients in urban and rural areas. Two of the three, Kansas City and Albuquerque, are the largest in their respective states. The similarities between all three are as remarkable as the differences.

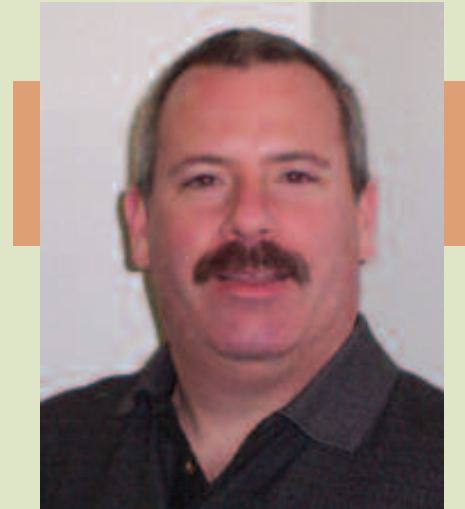

USING MMA TO LAUNCH A ZERO-BASED PRACTICE REVIEV IN KANSAS CITY

John E. Hennessy, CMPE, Executive Director

Kansas City Cancer Centers

Kansas City Cancer

Centers (KCCC) is a $37-$ physician practice that includes such services as medical and radiation oncology, blood and marrow transplant, and diagnostic imaging. The practice saw more than 9,800 new patients in 2004 from more than 40 counties in Kansas and 50 counties in Missouri. The payer mix is approximately $60 \%$ commercial and 40\% Medicare and Medicare Advantage and other government payers.

The practice is managed by John E. Hennessy, CMPE (Certified Medical Practice Executive). He and his physicians and managers concluded early in 2004 that MMA was a "done deal," and began planning for it a full year before it became law. By March 2004, the KCCC had developed and implemented a set of clinical pathways designed to define best practices for delivering chemotherapy by disease type, stage, and line of care.

\section{Clinical Pathways Point the Way}

These pathways, which are proprietary to the practice, are represented on a sheet of paper, as well as being available as a PDF document. For each disease, its stage, and line of care, there is a regimen of choice, backed up by what the practice considers to be the best science. At each stopping point, there is also a clinical trial of first choice. "By making a clinical trial the first choice in each case, we've also gotten increased clinical trial participation," says Hennessy. For the first time, KCCC is in the black in clinical trials more often than not, which was rarely the case before May of last year.

According to Hennessy, the pathways are an attempt to develop consistent "best practices" and reduce some of the random variation any practice might have with more than 30 oncologists treating different cancers. "Instead of having eight different ways of treating first-line lung cancer, we now have one or two," said Hennessy. "There also used to be a lot of confusion among nurses trying to figure out orders, but no more."

\footnotetext{
"INSTEAD OF HAVING EIGHT DIFFERENT WAYS OF TREATING FIRST-LINE LUNG CANCER, WE NOW HAVE ONE OR TWO."
}

- JOHN HENNESSY 


\section{Dramatic Drop in Drug Costs}

A recent evaluation showed what Hennessy calls a "dramatic" drop in drug costs. He also noted that patient care has been improved in a way that is both clinically and economically sound.

In the last 6 months of 2004, the practice also became aggressive in renegotiating managed care contracts. The oncologists wanted to preserve the best aspects of 2004, namely the increased administration codes, while locking in the average wholesale price of 2004 as a baseline for payments in future years. The practice was successful in negotiating three major payer agreements that met these criteria, and extended these terms through 2007.

The "re-engineering" of KCCC was not limited to process improvement, said Hennessy. It actually included redefining organizational values. "We went from a culture of 'me' to a culture of 'we', and from me-think to groupthink. The distinction is important because many group practices are physicians 'practicing alone together."'

Hennessy said the change has actually increased physician and staff satisfaction with the practice of oncology.

\section{Pull the Cord and Stop the Line}

Power was also delegated to non-physician employees such as financial counselors, nurses, and pharmacists. "These people are now empowered to pull the cord and stop the production line, if you will, if things aren't going right," said Hennessy. For example, if there are not required pre-certifications or if there are compendia listing issues identified. The traditional group practice prefers more centralized decision making.

Hennessy says that KCCC is now comfortable with the coding changes, although he characterizes them as a moving target. The first round did not get paid, but the firm has changed its rules, and he is confident they will be paid.

\section{Re-Engineering Minimized the Impact of Change}

There was no masking the financial impact of MMA: "Clearly, we've taken a huge revenue hit," said Hennessy. But re-engineering, process improvement, and cost containment have softened the impact. "We haven't been put in the position to look at programs and say we can't do this or that any more because we can't afford to," he explained.

The future? For his practice group, Hennessy forecasts more consolidation. KCCC just merged with a group of five radiation oncologists, and by April 2005, they will have added a second PET/CT scanner.

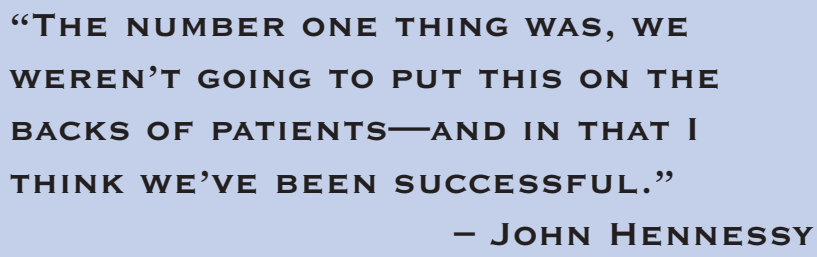

"A month into this, I don't know that anybody can say they'll thrive, but we're as well positioned as you can be. The number one thing was, we weren't going to put this on the backs of patients - and in that I think we've been successful," said Hennessy. "Quite frankly, the things we have done would have been healthy for the practice and the community regardless of whether the MMA changes existed."

Hennessy adds that they are being responsible custodians of resources, whether these be drugs or imaging equipment, and he believes that managed care companies and Medicare should acknowledge and encourage their responsible practices, rather than trying to knock prices down further or re-engineer the delivery system.

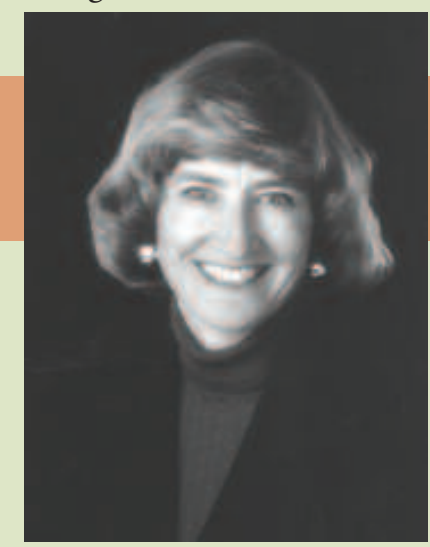

NEW MEXICO'S LARGEST PRACTICE FINDS A WAY TO KEEP TREATING THE INDIGENT
Barbara McAneny, MD, CEO New Mexico Cancer Center

New Mexico leads the country in both beautiful scenery and in poverty; it is one of the nation's poorest states. This presents special challenges for the state's largest oncology practice, the New Mexico Cancer Center (NMCC), and its CEO, Barbara McAneny, MD. Nevertheless, the practice has weathered MMA without either compromising care or curtailing expansion. JOP sought out Dr. McAneny and asked about her strategies and tactics.

First, some background: NMCC is headquartered in Albuquerque, the state's largest city, with a population of 500,000 . The NMCC treats 3,000 new patients each year, either in Albuquerque or at one of three satellite clinics. The practice employs eight medical oncologists, three radiation oncologists, and 120 support employees. 
The payer mix reflects the state: New Mexico has 26\% uninsured, and another $26 \%$ are either on Medicare or Medicaid. The remainder are managed care. "It's a tough market," says Dr. McAneny. She personally divides her time between practice management and patient care.

FIFTY-TWO PERCENT OF NEW MEXICO'S POPULATION ARE MEdICARE, MEdICAID, OR UNINSURED. "IT'S A TOUGH

MARKET.",

- BARBARA MCANENy, MD

Nevertheless, the practice is growing. It is in the process of developing a clinic on the Indian reservation in Gallup, scheduled to open this November. Extreme poverty is widespread on the reservations, and Dr. McAneny takes pride in the fact that NMCC has never turned away a patient for lack of ability to pay. She is quite up front about cost shifting from the drug mark-up, and says openly that there is no other choice. Among the practice's Medicare patients, 20\% have no Medi-Gap coverage, so the practice receives only $80 \%$ reimbursement. Instead of the average sales price plus $6 \%($ ASP +6$)$, the practice receives $86 \%$ of ASP from these Medicare patients.

"Our margins are exceedingly slim, and frankly challenge our ability to continue to provide care to these patients," said Dr. McAneny.

\section{Demonstration Project "A Lifesaver"}

The demonstration project has been a lifesaver, according to Dr. McAneny, by allowing the practice to both collect data and make ends meet for delivering in-office care.

She observed that the infusion codes for 2004 and 2005 almost meet the costs of infusions, but not quite: they cover about $90 \%$. Nevertheless, Dr. McAneny is happy to have them. "Without the new infusion codes, I don't know what we would be doing with either the uninsured

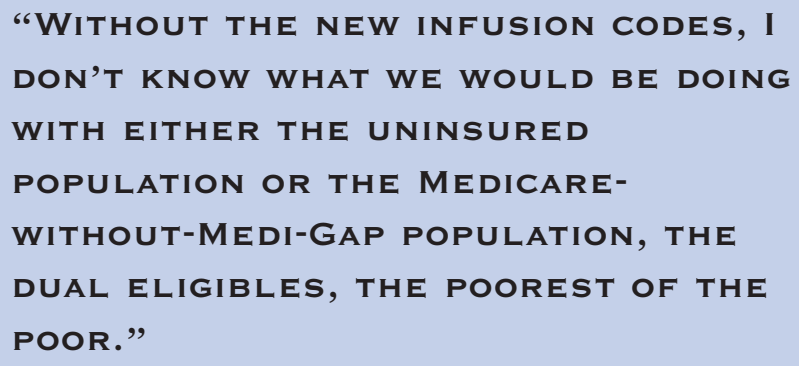

- BARBARA MCANENY, MD population or the Medicare-without-Medi-Gap population, the dual eligibles, the poorest of the poor."

NMCC still shifts costs from managed care contracts to take care of Medicare and the uninsured, but according to Dr. McAneny, this practice is not what it once was. "It gets tighter and tighter," she said. To ensure the overall health of the practice, McAneny offers the combination of medical oncology, diagnostic imaging, and radiation oncology, with an active clinical trials department. Overall, she is optimistic.

"The practice as a whole will continue just fine. And it is our goal to continue taking care of our Medicare and Medicaid patients, the folks without the $20 \%$ co-pay. It will be tight, but we intend to continue doing it," said McAneny.

\section{Forming a Viable Strategic Plan}

Strategic planning has been key. Planning for MMA was begun in 1999 , and by 2000 , the practice was already fully converted to electronic records. Along with streamlining operations, these efficiencies facilitate cost accounting, making it relatively easy to forecast the incremental costs of treating any patient. This data stream gives the practice more timely and accurate financial information.

Other efficiencies included hiring a full-time CPA, a human resources manager, an office manager, and two physician's assistants. All of the practice's physicians are required to be board-certified, and nurses are strongly encouraged to be certified by the Oncology Nursing Society (ONS).

One employee now works almost full-time getting replacement or "free drugs" from pharmaceutical makers through patient assistant programs. "We're very nervous about what's going to happen with these programs, because that's how we've been supplying a lot of people with chemotherapy," said Dr. McAneny. "It's easy for me to give away my time to somebody who can't pay me anything, but it's very difficult for me to buy them $\$ 30,000$ worth of chemotherapy."

\section{Coding Changes: A Source Of Confusion}

Coding changes were a source of confusion to everyone, including the carriers, Dr. McAneny noted.

"We'd send in the codes and they would send them back, saying we needed a modifier. We'd send them back with a modifier, and they'd say, 'Why did you do that?"' The practice is just now beginning to see payment for codes submitted early in January. Knowing cash flow would be a problem, they budgeted for it, even though that imposed a financial burden. Cash reserves are taxable. 
The practice maintains an active clinical trials department, and Dr. McAneny is happy when trials break even. "I look at that department as one of the costs of being a good oncologist. It's just part of the price of doing medicine. You've got to do it. I hope we can continue doing it. They do not make us money," she said.

\section{The Hidden Penalty of Clinical Trials}

Dr. McAneny no longer feels she can ethically offer trials to patients on the Medicare Advantage Program. When patients sign up for a trial, the MMA takes them off Medicare Advantage and puts them into Fee for Service Medicare. Patients on Medicare Advantage drop Medi-Gap. MMA argues that the Medicare Advantage plan is no longer responsible for the trial, meaning that when the patient joins the trial, he or she will suddenly be required to pay $20 \%$ of the cost of the drug-which they obviously cannot do. "The patient signed up for the HMO thinking they were getting rid of their co-pay!" says Dr. McAneny. "It doesn't seem ethical to offer that to a patient on Medicare Advantage."

\section{Working For Positive Change}

Dr. McAneny is intent that her practice will survive, but she is less optimistic about small practices, or those who did not plan as assiduously as NMCC. "MMA is just one more indication of how broken the health care system is. It was a rapidly conceived bill with a lot of flaws. Medicare has been around for 40 years; I didn't understand why it had to be reviewed and revised in a week."

However, as ASCO's AMA delegate, McAneny is working on positive change. "The only way physicians will have any hope of fixing the health care system is through organized medicine, through groups like ASCO and the AMA. When Congress decides to fix health care, they're not going to call up some individual physician — they're going to look to the groups."

Meanwhile, NMCC's Gallup expansion moves forward. Gallup is a poor city on a reservation that is a 2-hour drive from Albuquerque. This clinic is needed because patients were not getting full treatment. "They would be treated halfway through a course of radiation, and decide that they didn't want to be in Albuquerque any more. They would simply go home to the reservation, and that's unacceptable," said Dr. McAneny. "It may be foolhardy to expand in the face of the MMA changes, but I feel obliged to."

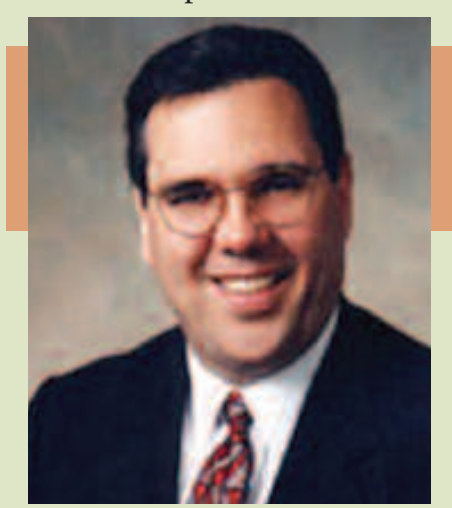

\section{TREATING 65 Patients OUt OF A CHEVY SUBURBAN}

Charles F. Winkler, MD, President

Purchase Cancer Group

Purchase Cancer Group of Paducah, Kentucky is a three-physician practice that operates four satellite clinics and a program for rural care. It cares for rural patients in western Kentucky and northwestern Tennessee, and to accomplish this, employs a staff of 36 . The staff includes eight oncology nurses and a licensed medical technologist. The Purchase Group is managed by Charles F. Winkler, MD, one of the three oncologists, with assistance from a practice manager.

The main clinic in Paducah sees about 250 patients per week, and the combined satellite clinics and rural care see about 350 more. The practice accepts about 900 new patients each year.

\section{Treating 65 Patients Out of a Chevy Suburban}

Four days a week, a Chevrolet Suburban leaves Paducah early in the morning carrying an oncologist, two or three oncology nurses, administrative staff, and all necessary chemotherapy. It stays out until all treatments are completed, typically seeing 60 to 65 patients per day. This mobile practice provides treatment, as well as $\mathrm{CBCs}$ and growth factors, just like the headquarters clinic.

\section{Building the Practice, but Increasing Hospital Referrals}

Knowing that MMA was coming, Dr. Winkler costed out every single protocol by June 2004. This led the practice to immediately eliminate Medicaid and Medicare without co-pay. They began referring these patients to the local hospital.

"Medicaid had always been something we couldn't do, but Medicare without a co-insurance we would sometimes do in

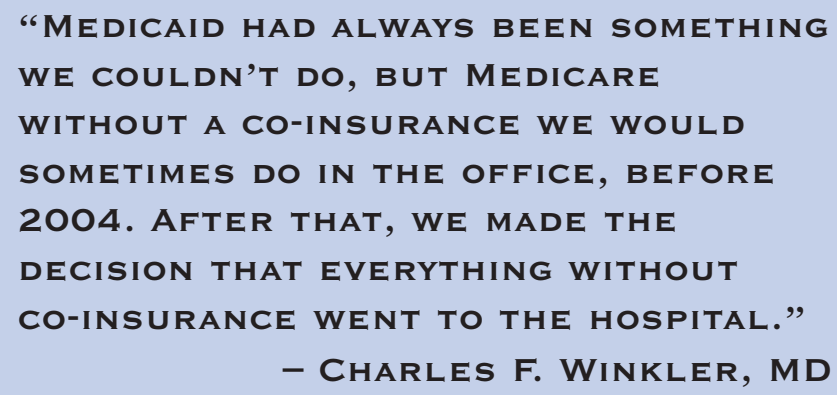


the office, before 2004. After that, we made the decision that everything without co-insurance went to the hospital," said Dr. Winkler.

Next, he and his colleagues took a hard look at regimens, and realized that some were simply too expensive. "We now have an insurance consult on every patient," said Dr. Winkler the result of this being that some patients are sent to the hospital. He is very direct: "It was a business decision based on money."

Dr. Winkler hastened to add that patients were not being disadvantaged. "I'm going to give them the same drug, but I'll give it to them in the hospital, not in the office."

\section{Hospital Referrals Less Than Ideal}

Prior to 2004, Dr. Winkler says there was no such thing as out-patient chemotherapy in the local hospitals.

Patients would simply be admitted to a bed on the floor. Then the hospitals designated some patient rooms as outpatient, but the treatment was less than ideal. It was done by an oncology nurse who had to take care of the entire floor, seeing both out-patients and in-patients. Sometimes a patient could not get a timely bed or timely treatment. "You could give this treatment in an hour and a half in the office, but in the hospital it would take 5, 6 hours," said Dr. Winkler.

How long hospital referrals can continue is open to question. According to Dr. Winkler, the "hospitals are losing their shirts." He concedes that this patient referral is, in effect, a form of cost shifting, burdening the hospitals. The hospital in Union City, Tennessee, has already stopped giving out-patient chemotherapy. "That's going to happen around the country," Dr. Winkler forecasts.

However, 2004 was a good year for the Purchase Cancer Group. "We made some business decisions that allowed us to continue oncology service to our patients," said Dr. Winkler. For some, this required hospital admission; for others, not.

According to Dr. Winkler, the forecast for 2005 is not clear. "We have seen a reduction of $6 \%$ for the month of January. But, we've had payers who didn't have the codes in and we've had tons of re-files. The clearing house we use for our computer system kicked out all our claims because we didn't have the demonstration project G-codes loaded," said Dr. Winkler. "We've had to drop everything to paper and refile. So the $6 \%$ reduction may actually be a fluke."

\section{Giving Away Treatments Was No Longer Viable}

As for tightening up operations, he says it was just good business practice. "We'd been giving away treatments, and it was no longer viable. We're a small practice, but we see lots of patients, and we buy several million dollars worth of drugs every year. We have to run it like a business," said Dr. Winkler. "Oncology in general used to have a fairly good margin, which allowed physicians to offset the underpayment for cognitive, management, and administration services. Now it's more like a grocery store, with reimbursement hovering from below cost to just slightly above cost on everything. If you don't watch your pennies, you're going to lose your shirt," he said.

The Purchase Group also converted to a paperless office, and now they scan everything into a document management system. They communicate by the Internet. Dr. Winkler is currently looking into voice-recognition dictation to save on transcription costs. All laboratory work has been consolidated into the main office, and is managed as a revenue stream.

The Demonstration Project has been helpful, said Dr. Winkler, but he suspects it may be finite. "The thing they're going to get you on is if you just keep dictating the same thing over and over again. You've got to put something in there that you made a conscious decision to make something better." The other side of the coin, he says, is if oncologists identify that they are managing side effects, then they should be paid more.

It has been difficult for the Purchase Group to participate in clinical trials because of the dedication and manpower required. Trials also require a dedicated nurse. ASCO's published data shows break-even at best for trials, and Dr. Winkler agrees. "For the time intensity a clinical trial requires, it was just not worth it for us. But," he says, "we're in a paradigm shift, and we have to look at everything again. Maybe we'll take another look at trials, too."

Dr. Winkler is cautiously optimistic about the future, but if he is proven wrong, says he is prepared to merge with another oncology practice. In that case, he would take early retirement and work as a locom tenens.

He is philosophical. "Your whole job, what you really want to do, is just take care of patients," he said.

\section{Across the Three Practices, More Similarities Than Differences}

Despite size differences, all three practices have managed not only to survive, but to thrive in the wake of the largest oncology business change in recent memory. What they have in common are medical excellence and astute business skills.

Certain subtle commonalities have also emerged. All three are diversified, and all three have streamlined 
operations and installed "best practices" of doing business to allow them to focus on patient care. Even the smallest practice continues to pursue efficiencies such as conversion to the paperless office with voice recognition technology. In the wake of MMA, business efficiencies are no longer a luxury.
And yet, none of the three has compromised patient care. The medium-sized practice has an employee working almost full time to obtain replacement of free drugs.

All three practices keep an open mind toward expansion, diversification, or merger, if need be.

\section{Proposed Rule Published for Competitive}

ACQUISITION PROGRAM

The Centers for Medicare and Medicaid services (CMS) have issued a proposed rule to implement a competitive acquisition program (CAP) for Part B drugs as required by the Medicare Modernization Act of 2003. This program will become available for services on or after January 1, 2006. The CAP program will be phased in, although CMS has not yet determined the phase-in methodology.

Under the proposed rule, physicians will annually elect whether to participate in the CAP and obtain all of their drugs for Medicare patients under the CAP, or continue to purchase drugs from distributors or manufacturers and seek reimbursement directly from CMS. Physicians who elect to participate in the CAP program will choose a vendor to be their sole source for the selected categories of Part B drugs. Physicians will continue to bill CMS for drug administration services and will receive the same Medicare payment for administration as physicians who purchase drugs in the marketplace.

Although CMS states that obtaining drugs from a participating vendor will be "simple and efficient," the proposed rule includes several issues that may create additional administrative burdens for oncologists. For example, physicians receiving drugs from the vendor will be required to keep a separate paper or electronic inventory record for each drug and report to the carrier a prescription number assigned by the vendor as each drug is administered. Additionally, the method for dealing with unused drugs will require additional administrative effort as outlined in the proposed rule. The physician and vendor are required to negotiate with respect to the disposition of the unused drug, and if the drug is retained for a future patient, the physician would submit a new order with respect to the new patient, but note in the order that a new drug is not required.

The proposed rule seeks public comment on key elements of the CAP program including: the selection of categories of drugs to be available through the CAP program; whether categories should be phased into the CAP; and standards for vendor selection. A final rule will be published by CMS later this year. 